\title{
Deep axillary lymphadenopathy after coronavirus disease 2019 vaccination: a case report
}

\author{
Mio Mori ${ }^{1} \cdot$ Tomoyuki Fujioka $^{1}$ [D $\cdot$ Yuka Yashima $^{1} \cdot$ Emi Yamaga $^{1} \cdot$ Tsuyoshi Nakagawa $^{2} \cdot$ Kazunori Kubota $^{3}$. \\ Ukihide Tateishi ${ }^{1}$
}

Received: 15 April 2021 / Accepted: 28 July 2021 / Published online: 25 October 2021

(c) The Author(s), under exclusive licence to The Japan Society of Ultrasonics in Medicine 2021

We present a case of axillary lymphadenopathy that occurred after COVID-19 vaccination and that mimicked metastasis in a Japanese woman in her $30 \mathrm{~s}$. There was no lymphadenopathy on her ultrasonography (LOGIQ E10s, GE healthcare Japan, Tokyo, Japan) for breast cancer screening performed on the same day as the administration of the Pfizer-BioNTech vaccine (Fig. 1a). This was the first time she had been vaccinated against COVID-19. Nine days later, she noticed painful axillary masses and we found lymphadenopathy in her axilla on ultrasonography. The largest of her swollen lymph nodes was deep in level I, and the size was $20 \times 15 \mathrm{~mm}$ (Fig. 1b). The lymph nodes at the superficial axilla were up to $12 \times 7 \mathrm{~mm}$ (Fig. 1c). In a follow-up ultrasonography 14 days after the vaccination, the lymph nodes shrank slightly (Fig. 1d), and we determined their swelling to be reactive.

There have been several studies reporting axillary lymphadenopathy after vaccination [1-4], and these have reported multiple lymphadenopathies on the ipsilateral side of the vaccination. This phenomenon has been reported to be more common in the those aged $<64$ years [3]. Given the COVID-19 vaccines provoke a highly immunogenic clinical response in patients, it is reasonable to expect swelling of multiple lymph nodes instead of a single one [4]. The vaccine is usually injected into the deltoid muscle, suggesting that the localization of lymphadenopathy has anatomical features distinct from breast cancer metastasis. The sentinel lymph node of breast cancer is usually located at the axillary fat pad on the lateral of the pectoralis major muscle, and multiple lymph node metastases are often concentrated in the sentinel lymph node [5]. In our case, the largest lymph node was deeper than the lower edge of the pectoralis major muscle. Although not explicitly mentioned, several reports have presented lymph nodes at the same site [2, 4], which is perhaps due to the different lymphatic flow between the breast and the upper arm. We found it difficult to distinguish between benign and malignant swelling based on morphology, given we found distorted cortical thickening in our case (Fig. 1b) and we often observe smooth lymph node metastases.

Reactive axillary lymphadenopathy following COVID-19 vaccination might mimic metastasis. Therefore, radiologists/ clinicians should assess the recent history of COVID-19 vaccination in cases of unilateral multiple axillary lymphadenopathies in deep axilla.
Tomoyuki Fujioka

tomoyukifujioka@hotmail.com

1 Department of Diagnostic Radiology, Tokyo Medical and Dental University, 1-5-45 Yushima, Bunkyo-ku, Tokyo 113-8510, Japan

2 Department of Surgery, Breast Surgery, Tokyo Medical and Dental University, 1-5-45 Yushima, Bunkyo-ku, Tokyo 113-8510, Japan

3 Department of Radiology, Dokkyo Medical University Saitama Medical Center, 2-1-50 Minamikoshigaya, Koshigaya, Saitama 343-8555, Japan 
Fig. 1 A woman in her $30 \mathrm{~s}$ with no medical antecedents. a The normal axillary lymph nodes on screening ultrasonography (arrows). b Nine days later, targeted sonography revealed a left axillary lymph node measuring $20 \times 15 \mathrm{~mm}$ (arrow) deeper than the lower edge of the pectoralis major muscle. c Lymph nodes up to $12 \times 7 \mathrm{~mm}$ were found on the front side of the lower edge of the pectoralis major muscle (arrow). d In a follow-up ultrasonography 14 days after vaccination, the lymph nodes shrank slightly (arrows). Arrowheads, the hyperechoic line of the lower edge of the pectoralis major muscle; " the pectoralis major muscle

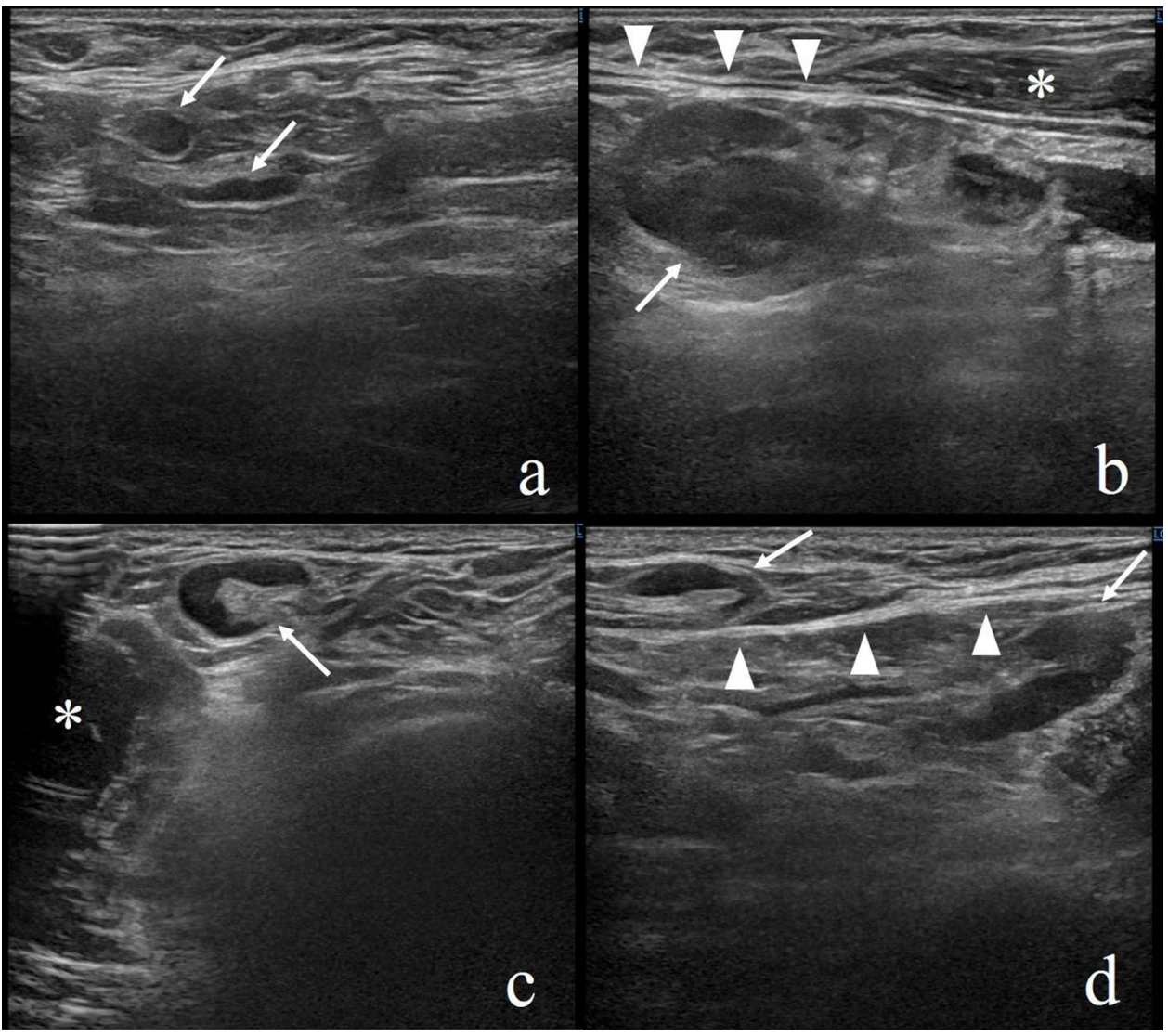

Author contributions Conceptualization - TF; Data curation - YY, EY, TN; Formal analysis - None; Funding acquisition - None; Investigation - MM, YY, EY, TN; Methodology - TF, KK; Project administration TF; Resources - UT; Software - None; Supervision - TF, KK; Validation - TF, KK; Visualization - MM; Writing - MM; Writing - review and editing - MM, FT, KK, UT.

\section{Source of funding None.}

\section{Declarations}

Conflict of interest We declare that there are no conflicts of interest.

Ethical statements All procedures followed were in accordance with the ethical standards of the responsible committee on human experimentation (institutional and national) and with the Helsinki Declaration of 1964 and later versions.

Informed consent Informed consent was obtained from the patient for being included in the study.

\section{References}

1. Edmonds CE, Zuckerman SP, Conant EF. Management of unilateral axillary lymphadenopathy detected on breast MRI in the era of coronavirus disease (COVID-19) vaccination. Am J Roentgenol. 2021;217:831-4.

2. Mehta N, Sales RM, Babagbemi K, et al. Unilateral axillary adenopathy in the setting of COVID-19 vaccine. Clin Imaging. 2021;75:12-5.

3. Centers for Disease Control and Prevention. The moderna COVID19 vaccine's local reactions, systemic reactions, adverse events, and serious adverse events. [serial on the Internet]. 2021 [cited 29 Sept 2021]. Available from: https://www.cdc.gov/vaccines/covid19/info-by-product/moderna/reactogenicity.html.

4. Lehman CD, D'Alessandro HA, Mendoza DP, et al. Unilateral lymphadenopathy after COVID-19 vaccination: a practical management plan for radiologists across specialties. J Am Coll Radiol. 2021;18:843-52.

5. Lo C, Lee PC, Yen RF, et al. Most frequent location of the sentinel lymph nodes. Asian J Surg. 2014;37:125-9.

Publisher's Note Springer Nature remains neutral with regard to jurisdictional claims in published maps and institutional affiliations. 